\title{
Elementos de la gestión organizacional: la racionalidad instrumental y sus límites. Algunos modelos explicativos
}

\author{
Marcelo Sánchez-Oro Sánchez \\ Universidad de Extremadura \\ msanoro@unex.es \\ José Antonio Pérez Rubio \\ Universidad de Extremadura \\ joseantonioperezrubio@gmail.com \\ Héctor Valentín Jiménez Naranjo \\ Universidad de Extremadura \\ hectorjimenez@unex.es
}

Resumen: Este artículo trata de los problemas de gestión administrativa y su relación con la racionalidad instrumental en las organizaciones, especialmente las empresas. A partir de la crítica a la racionalidad como ideal tipo, se observa que la existencia de diversas interpretaciones de la conducta racional en las organizaciones productivas, tanto en la administración de las cosas como de las personas, deberían ser el paradigma de la lógica del sistema capitalista. Desde diferentes puntos de vista, teniendo en cuenta los factores internos y externos, se suele dar una explicación que justifica la acción racional en la persecución de los objetivos y su acción cambiante. Se trata de un intento de aproximación a los diferentes órdenes que se promocionan al socaire de los cambios $y$ las «modas» intelectuales, tratando de dar satisfacción a la curiosidad cientifica sobre cuestiones epistemológicas relacionadas con las orientaciones que condicionan las organizaciones dentro del sistema socioeconómico dominante.

Palabras clave: sociología organizacional; racionalidad limitada; organizaciones; empresas. 
Elements of organizational management: instrumental rationality and its limits. Some explanatory models

\begin{abstract}
This article deals with the problems of administrative management and its relation to instrumental rationality in organizations, especially companies. Based on the criticism of rationality as an ideal type, it is observed that the existence of diverse interpretations of rational behavior in productive organizations, both in the administration of things and of people, should be a paradigm of the capitalist system. From different points of view, taking into account the internal and external factors, an explanation is usually given that justifies rational action in the pursuit of objectives and changing actions. It is an attempt to approach the different orders that are promoted in the wake of changes and intellectual "fashions" by trying to satisfy scientific curiosity on epistemological issues related to the orientations that condition organizations within the dominant socioeconomic system.
\end{abstract}

Keywords: organizational sociology; limited rationality; organizations; business 


\section{Gestión organizacional y racionalidad administrativa}

El asentamiento de los principios de la racionalidad y su extensión preocupa a los técnicos e investigadores de los problemas que condicionan el desarrollo de las organizaciones dentro de los sistemas socioeconómicos. Se podría decir que persiste la idea de lo económico como esfera autónoma, lo cual, a nuestro juicio, refleja la visión dominante en diferentes ámbitos académicos e institucionales. Sin embargo, el avance de la «racionalidad» supone la ampliación, al campo de las relaciones sociales, de los criterios de la organización mercantil. Desde ese momento, explica Furtado (1982), aparece el proceso de subordinación del proceso social a los criterios de «racionalidad instrumental» y las consecuentes modificaciones dentro de las estructuras sociales.

Este proceso no deja de tener su idiosincrasia en función de los condicionantes y factores históricos que lo acompañan. Dicho de otro modo, la originalidad de Schumpeter reside en la idea de que, si bien el empresario capitalista se imbuía de un clima racional general sus manifestaciones, a partir del desarrollo de la moneda, de la ciencia moderna y de la libertad individual, sin embargo, de ninguna manera constituía la personalidad modélica de la cultura capitalista (Martinelli, 1985). La acción innovadora respondería a una racionalidad que no se corresponde con el cálculo de la propia utilidad, sino con una racionalidad entendida como la capacidad de imaginar, de actuar y crear algo nuevo. Esta concepción presenta una notable originalidad, no solo respecto a la tradición clásica y neoclásica del pensamiento económico (del homo ceconomicus), sino también respecto a las contribuciones sociológicas de la teoría de la acción y del análisis del capitalismo que formulan Weber, Sombart y Pareto. Efectivamente, en los tres, aunque en forma y grado diferentes, existe una tendencia común a caracterizar la sociedad industrial capitalista mediante la progresiva institucionalización de una mentalidad económica (o «espíritu del capitalismo») y la difusión de un modelo de comportamiento que resulta, en conjunto, racionalista, individualista y adquisitivo. Sombart opone a estas características otras orientaciones de tipo tradicional, solidario y de simple satisfacción de la moralidad que él entiende representativa de las sociedades preindustriales. Max Weber, por su parte, descubre en la racionalidad orientada a un fin el «tipo ideal» de desarrollo económico, y en la autoridad racional-legal, el «tipo ideal» de autoridad, entendidos como propios del capitalismo moderno. A su vez, Wilfredo Pareto encuentra, en la mayor parte de la actividad económica, un modélico campo de aplicación de las acciones lógicas. Si bien estos autores no reducen la acción del empresariado capitalista al móvil utilitarista, tienden a ver en los valores y actitudes racionalistas, individualistas y utilitaristas los elementos de un síndrome cultural unitario, y también a acen- 
tuar la especificidad de este síndrome y de esta forma de comportamiento social, que aparecen aquí como construcciones analíticas. Schumpeter, señala Martinelli (1985), subraya sobre todo la coexistencia de elementos pre-y postindustriales, de modo que el éxito del empresario se debe a su capacidad para aplicar la energía vital del líder preindustrial al contexto más favorable del capitalismo racionalista.

No obstante, a partir de Weber y su estudio de la burocracia, se pondrá en valor una de las características más sobresalientes del comportamiento administrativo, cuando se la compara con el comportamiento humano en otros contextos: en la medida en que las organizaciones son sistemas orientados a la realización de unos fines, el comportamiento de una organización busca pautas eficaces de actividad que estén orientadas a los fines señalados, e influir sobre las personas adscritas a la misma para que adopten dichas pautas. Efectivamente, el principio epistemológico weberiano se asienta en establecer observaciones causales específicas de una naturaleza general que asume un carácter de cuasi ley (Jiménez Blanco, 1978).

Las organizaciones, su estructura, su dinámica y su gestión son parte esencial de la producción sociológica, que recoge e integra las contribuciones de otras áreas (antropológicas, psicológicas, políticas y económicas) y ha impulsado el desarrollo de la sociología en su conjunto (Rodríguez, 1991: 203; Lucas Marín y García Ruiz, 2002; González, 2003; Bude, 2017). Aunque la investigación de las variables motivacionales ha tenido en el área de la sociología un mayor desarrollo en la construcción de modelos empíricos de explicación (Pérez Rubio, 1997).

La sociedad industrial ha sido denominada como una sociedad organizacional y a quien en ella interactúa se le ha llegado a calificar de «hombre de la organización» (López Pintor, 1986). La vida del actual transcurre en el seno de organizaciones complejas (sistema escolar, empresas, asociaciones profesionales, etc.). El interés de la sociología por el fenómeno organizacional se ha localizado inicialmente en la organización industrial. Por esta razón, la perspectiva racionalista weberiana debe ser adecuadamente dimensionada, y para ello es necesario poner su concepción de la racionalidad de las organizaciones en relación con el conjunto de teorías que se han ido desarrollando. Este corpus teórico evoluciona a través de dos ejes: el binomio sistema abierto/sistema cerrado y la perspectiva racional/perspectiva social (Bauman, 2003). La perspectiva sistémica de análisis está presente en todas las concepciones teóricas, la variación se sitúa en la mayor o menor apertura del sistema. La perspectiva racional considera que la estructura de la organización es un instrumento para conseguir unos objetivos determinados, lo que supone implícitamente dar prioridad a los elementos formales (coordinación, planificación, etc.), sobre aquellos que se fundamentan en los miembros de la organización (satisfacción, motivación, etc.). Por el contrario, la perspectiva 
social hace hincapié en el conflicto y la lucha por el poder (Veroz Herradón, 1999: 28 y ss.; Bauman, 2003).

A tenor de esto, nuestro objetivo con este artículo es señalar cómo en la gestión organizativa, la racionalidad, a partir de la crítica al ideal tipo weberiano, ha ido evolucionando al considerar que aquella debe adaptarse a las «turbulencias», tanto internas como externas. Dado que, como indican Brunet Icart y Catalin Mara (2018), las instituciones no se reducen a reglas de juego que los individuos deciden en función de si les son útiles para sus intereses, como afirma la teoría de la elección racional, sino que son la realidad social, una trama compleja de imposiciones, de relaciones sociales impuestas que existen y cambian en la historia. Ello exige volver a pensar los conceptos que han servido para explicar la acción y determinar su validez a la hora de interpretar las transformaciones experimentadas en las últimas décadas en todas las facetas de nuestra sociedad, incluidas las estructuras de sus organizaciones.

A efecto ilustrativo, como aproximación empírica basada en el «método del caso» (Villarreal Larrinaga, 2017), se expone como referencia de ejemplo del desplazamiento de objetivos y sus disfunciones la red de restaurantes de comida rápida McDonald's, y de la diversidad de formas de racionalidad en la toma de decisiones y de los sistemas productivos, la controversia fordismo postfordismo, ejemplarizada, en este último caso, en el productor japonés de vehículos Toyota. Estos ejemplos, como todos, son reduccionistas, y sirven solamente (aunque a veces ya es bastante) para ilustrar algunas de las situaciones que se explican en este artículo.

\section{Las formas de la racionalidad organizativa}

Las explicaciones relativas a los comportamientos y las acciones en las organizaciones se pueden dividir en dos corrientes (Delcourt, 1973). La corriente «racionalista», la cual explicaría la organización como un sistema programado con base en sus fines y objetivos, en la jerarquización y en la determinación de los medios más eficaces para poner su proyecto en marcha. Para esta corriente el funcionamiento de la empresa está cada vez mejor programado y su desarrollo, planificado. Alain Touraine (1973) apunta que, bajo este prisma, la sociedad y sus organizaciones están en un continuo desarrollo, en función de la aplicación de modelos racionalizadores. La corriente «realista» subraya que los objetivos reales de la organización pueden ser diferentes de los objetivos dados a conocer, es decir, frente a los objetivos declarados por la organización existen otros objetivos que responden a intereses de grupo. Estos objetivos evolucionan o cambian según los problemas que se plantean en función de las solidaridades y las alianzas en la or- 
ganización. La separación entre los objetivos declarados y los perseguidos por los grupos de interés corre el riesgo de ser cada vez mayor, a medida que el entorno exterior manifiesta una evolución rápida y agitada (imagen 1 ).

Imagen 1. Comportamiento organizacional

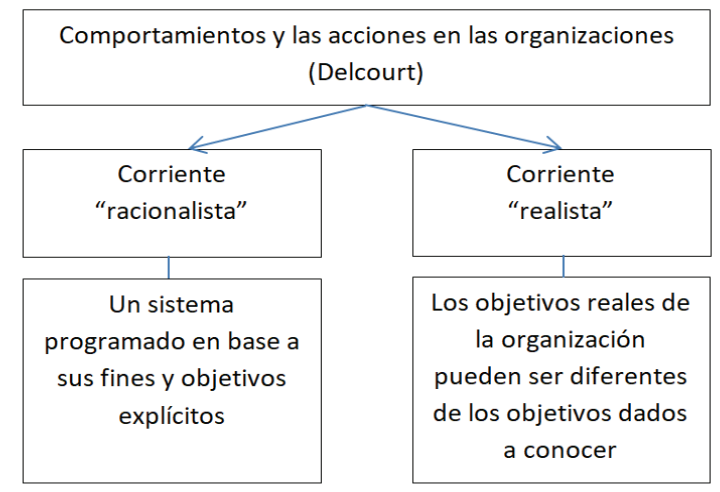

Fuente: elaboración propia a partir de Delcourt, 1973: 1.

En consecuencia, es posible afirmar que existen formas de racionalidad en las organizaciones, que no están articuladas por un sistema racional ligado necesariamente a aspectos técnicos y económicos. Por tanto, hay diversas formas de racionalidad con combinaciones y/o contradicciones posibles y que son alternativas a la corriente racionalista en lo que, paradójicamente, señala Weber, como la racionalización que produce resultados irracionales a medida que las burocracias adquieren vida propia y se expanden por toda la vida social. Giddens (2010), citando a Ritzer, explica este proceso a partir de la cadena de comida rápida McDonald's. Un sistema aparentemente racional de elaborar comida «genera una serie de irracionalidades», que dañan tanto nuestra salud como el medio ambiente. Pero lo interesante es considerar la diferente perspectiva con la que se evalúa el modelo productivo de racionalidad de la «macdonalización», según se trate de los clientes o de los empleados. Los primeros solo llegan a comer la imagen pública de McDonald's, perfectamente cuidada por intensas y extensa campaña de marketing. El cliente pensará que el restaurante es eficaz y bien organizado. Plenamente racional. Tal vez la perspectiva «realista» la tengan los empleados, más conscientes de la ineficiencia, las presiones externas e internas y los objetivos reales de la organización: conseguir el máximo beneficio; un tanto alejado de los valores y la misión oficialmente publicitada por la marca. La conclusión es obvia, para comprender a las organizaciones hay que investigar su planificación interna y sus prácticas de gestión, pero también el rol que cumplen en la sociedad. 
Max Weber distinguió la racionalidad sustantiva o social de la racionalidad formal o instrumental. La primera, referida a elección de fines, y la segunda, la referida a la elección de medios de relación a fines definidos. A partir de estos criterios se distinguen dos tipos de organización: aquellas cuya delimitación de objetivos se puede hacer desde la organización, como la empresa económica; o bien aquellas cuyos objetivos no se fijan por la organización sino por la instancia que las controla, como las organizaciones comandadas (la prisión, el ejército, etc.). La racionalidad social expresa los juicios de valor en la base de las acciones independientes de los costes para los individuos y las instancias. La racionalidad instrumental, también llamada pura o formal, es aplicable solamente en función de criterios definidos. La racionalidad instrumental determina lo factible, desde ella se analizan los medios para la realización de un fin determinado. Basada sobre normas y criterios, ella ayuda a determinar los medios más productivos, los más eficaces, que no tienen por qué ser los menos costosos. En resumidas cuentas, mientras que la racionalidad formal de la acción económica es indiferente al contenido - los valores y objetivos-, y las consecuencias de la acción — más allá del motivo de referencia de la acción-, la racionalidad material, en cambio, se interesa exclusivamente por el contenido que anima la acción y por sus consecuencias. El hecho es que la racionalidad formal y la racionalidad material están en tensión irresoluble, porque la racionalidad formal está siempre al servicio de los motivos y valores éticos-materiales, $y$, por consiguiente, tiene consecuencias de orden ético-material (López Novo, 1994) (imagen 2).

\section{Imagen 2. Racionalidad en Weber}

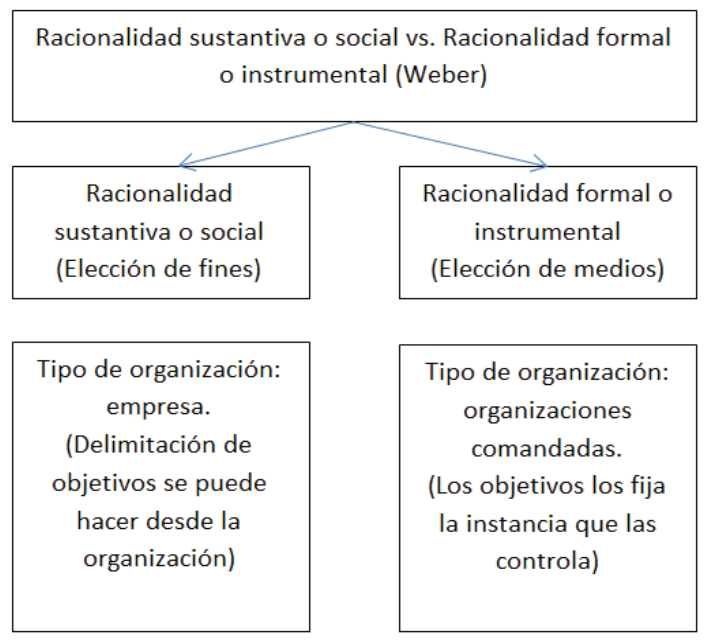

Fuente: elaboración propia a partir de López Novo, 1994: 39. 
Como ya se sabe, los criterios de la racionalidad instrumental toman como referencia tanto la componente técnica y de viabilidad como la previsión de comportamientos. La componente técnica supone que toda acción es operacional porque produce los efectos deseados. La vía técnicamente racional viene motivada por que las relaciones causa-efecto son fácilmente conocidas y controladas. Se admite que, a veces, los medios empleados no permiten más que una realización parcial del objetivo, o que puedan conducir a efectos no queridos o efectos inesperados, pudiendo repercutir sobre la definición y jerarquía de los objetivos. La componente de viabilidad y rentabilidad económica trata de la distribución y asignación de medios escasos para conseguir fines múltiples con objeto de optimizar los resultados. La tercera componente hay que centrarla en el plano organizacional. Si bien la racionalidad instrumental tiene por objetivo prioritario una administración de cosas lo más racional posible, no está separada del gobierno de los hombres, es decir, en la administración de las cosas no se pueden subestimar las reacciones posibles de parte de los miembros y grupos de interés. Una organización eficaz supone una coordinación y una previsión de comportamientos. La forma de organización racional sería aquella que permite colaborar y aceptar la participación en una tarea en la que es capaz de aplicar la racionalidad técnica y/o económica. La racionalidad burocrática sería un sistema de gestión equitativo e imparcial de las personas vinculadas, la cual les compromete en una colaboración eficaz en la consecución de los objetivos y a través de medios definidos. Subyacente a la racionalidad técnico-económica habría una racionalidad de la organización que asegura la orientación y el manejo de las personas. Pero, a medida que la racionalidad formal invade sectores de la vida en sociedad, el mundo se seculariza y tiende a desmitificarse, como señala Touraine (1965), comienza la desaparición de las garantías «metasociales». Así la organización burocrática aparece como superior y más eficaz porque permite el control y previsibilidad de los comportamientos. Esta previsibilidad obtenida por la estandarización es inseparable de la impersonalización y la deshumanización de las relaciones sociales (Mottez, 1971).

Por tanto, la racionalidad instrumental (Bilbao, 1996) no es una racionalidad parcial que se centra solo en el campo de las relaciones económicas. Precisamente, es la extensión de la racionalidad económica uno de los síntomas de la modernidad. Esta racionalidad se construye sobre la norma que rige el pensamiento científico: la centralidad del método. Lo característico de la economía es que se constituye como principio regulador de las relaciones entre los individuos. La modernidad se articula como una relación triangular. El primer punto es el despliegue paralelo entre la noción de individuo y racionalidad instrumental. El segundo es una perspectiva que pone el acento exclusivamente en la corrección del 
procedimiento. Lo decisivo, desde el punto de vista de la racionalidad económica, no son sus consecuencias sociales, sino el cumplimiento de sus supuestos. Las creaciones sociales que hacen viable la lógica del mercado pasan a ser función de la política. Una vez alcanzado este punto, la organización social se configura plenamente moderna. El último aspecto lo constituye el estatuto del individuo. La configuración de la economía como ciencia implicaba la sustitución del individuo real por su representación. La práctica de la racionalidad económica requiere la desmaterialización del individuo real y su homogeneización bajo la figura del individuo representado por la teoría (Rifkin, 2014; Beck, 2009; Simon, 2003).

\section{La quiebra de la racionalidad instrumental a partir de la multiplicidad de objetivos}

Si la racionalidad instrumental se presenta como la adaptación de medios técnicos, económicos y/o de organización a uno o varios fines predeterminados, entonces estos principios o criterios de racionalidad no podrán ser aplicables en el caso de que los fines y objetivos determinados no sean lo suficientemente concretizados y aceptados por las personas y los grupos responsables de una empresa o de una organización. Etzioni (1962) y Perrow (1975) destacaron que los objetivos son definidos a diferente nivel. Una primera distinción puede ser la que hay entre los objetivos oficiales propuestos por la organización y sus objetivos operacionales. Los objetivos oficiales son los que aparecen declarados a nivel del discurso de los actores en presencia (escrito o verbal) sobre lo que persigue o debe perseguir la organización. Los objetivos operacionales son los objetivos definidos concretamente en las políticas. Estos determinan las actividades concretas en el seno de la organización; estos objetivos indican lo que se hace separadamente o contrariamente a lo que se declara.

La corriente realista se opone a la corriente racionalista, sobre todo en que los objetivos declarados intervienen en una estrategia de legitimación de la organización en relación con su ámbito interno (los trabajadores y sus diversas agrupaciones) y externo (todos o parte de los clientes y terceros). En esta perspectiva, los objetivos oficiales deben ser analizados en función de los intereses que los dirigentes o ciertas partes de la organización tienen. Dicho de otro modo, tal como evidenció Merton (1957), existen objetivos inconfesables y ocultos en cualquier organización (Giddens, 2010).

La fijación de objetivos finales o intermedios no supone que aquellos no puedan ser contradictorios, de tal forma que la consecución de uno excluya al otro en el momento que estos estén jerarquizados. En toda organización, la racionalidad económica se encuentra cogida entre las condiciones determinantes de la eficacia 
económica interna y la externa, es decir, en condiciones cambiantes. Ahora bien, la corriente racionalista no solo subestima la dificultad de combinar los criterios de racionalidad, sino que también infravalora la dificultad de fijar objetivos concretos a corto plazo o a través del tiempo, es decir, en un entorno de condiciones que cambian constantemente. La corriente racionalista supone, en realidad, que el entorno no cambia, y una cierta rutina es factible en la prosecución de los objetivos gracias a un sistema de organización relativamente estable. Thompson (1967) señaló la necesidad de medir el grado de incertidumbre en toda acción, sobre todo económica. Para este analista, cuando los cambios se mantienen en ciertos límites, las organizaciones encuentran medidas para evitar la perturbación, construirán mecanismos amortiguadores o procesos que tienen como misión neutralizar el efecto de las variaciones. Thompson pone como ejemplo los siguientes: frente a un mercado irregular se trabajará con stocks; para evitar las averías o paradas de máquinas, se revisarán y se mantendrán los equipos; para evitar los efectos provocados por la diversidad de personas implicadas en una empresa, se las formará y adoctrinará, etc. La creación de «amortiguadores» en la organización supone tener sistemas de anticipación a las variaciones a partir del conocimiento de la «horquilla» donde aquellas se producen. Las adaptaciones serán posibles si los cambios en el entorno no son muy bruscos, ni muy importantes, y si el tiempo de reacción es corto. Estas condiciones de adaptación son difíciles ante un entorno complejo y acelerado (Delcourt, 1973).

Pero otra forma de anticiparse a las variaciones del entorno es el desplazamiento de objetivos. Aparte de la constatación del aspecto cambiante o evolutivo de los objetivos, los estudios de casos (hospitales, penitenciarías, etc.) demuestran la evidencia de que, además, estas organizaciones persiguen varios objetivos al mismo tiempo (Perrow, 1975). De esta forma, la corriente «realista» centra su atención en la definición del concepto de objetivo, dado que muchos de los estudios tienen por finalidad el análisis de estas organizaciones cuando cambian sus objetivos. Perrow señala que «lo que se designa como objetivo, así como lo que se usa como prueba de su existencia, depende en gran parte de la finalidad del estudio de que se trate». Decir, como afirma Parsons, que una organización se define como una entidad que persigue un objetivo específico es quizá tan cierto como la observación, mucho más frecuente, de que las organizaciones tienen múltiples objetivos (Perrow, 1975: 475). Incluso en el caso de las empresas, pueden tener como objetivo principal el incremento de productividad, pero un análisis más en detalle puede arrojar que hay otros objetivos tan importantes o más, como puede ser la conservación de la organización o como lo que descubrió Michels (1967), el mantenimiento de posiciones de prestigio y poder en la cúspide de la pirámide organizacional. Perrow llega a distinguir seis grupos o clases de objetivos, aun a 
sabiendas de que este número pudiera ser mayor: tres son de carácter o de referencia externa, que los denomina «objetivos de la sociedad», «de la producción» $\mathrm{y}$ «de los inversionistas». Y otros tres, de carácter o de referencia interna, que son los «objetivos del sistema» (supervivencia, crecimiento, etc.), «los objetivos del producto» (características que definen al producto, como la calidad, disponibilidad, el estilo) y «los del grupo», que denomina objetivos derivados, es decir, los de los individuos que hacen uso del poder de la propia organización para perseguir los demás objetivos (imagen 3).

\section{Imagen 3. Multiplicidad de objetivos}

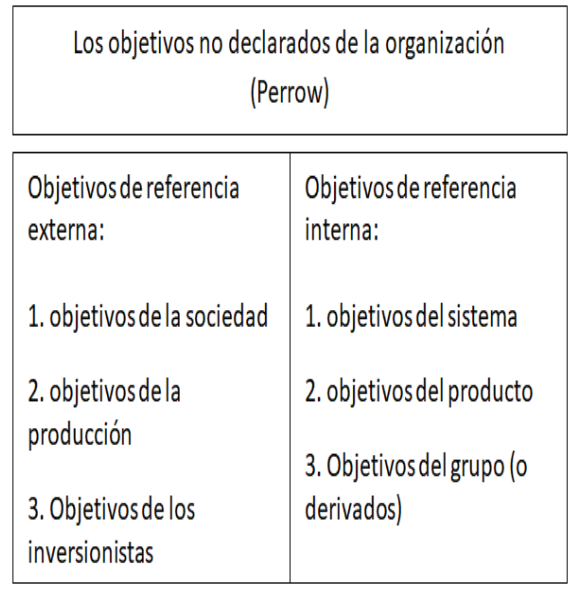

Fuente: elaboración propia a partir de Perrow.

La postura de Perrow no solo está en reconocer que las organizaciones pueden perseguir diversos objetivos, sino que dichos objetivos pueden entrar en competencia y, en muchos casos, pueden ser incompatibles.

A medida que crece la empresa, crecen los niveles, los departamentos, los servicios, los establecimientos o las redes, y, por ende, se generan numerosas e impersonales formas de relación. A medida que aumenta el número de individuos, más grupos diferentes se formarán y más difícil será conseguir la «lealtad», por lo que es necesario establecer reglas que aseguren la equidad o la imparcialidad en la selección, en el trato, así como en la objetividad de los juicios. Como decíamos, en la administración de los hombres son básicos la previsión de comportamiento, el establecimiento de derechos y deberes, coordinación de las tareas de individuos y los grupos. Esto supone un consenso de individuos y grupos sobre la legitimidad de las reglas y de las sanciones y recompensas asociadas a aquellas, una creencia 
en su racionalidad, su eficacia, su imparcialidad o equidad (este puede ser el caso de la remuneración, la promoción, etc.).

Sastre ilustra este problema, rescatando de nuevo a George Ritzer y su sociedad mcdonalizada. Los objetivos no declarados de las grandes organizaciones buscan la eficiencia organizativa y, lo que es más interesante, consumidores eficaces (Sastre, 2010). En los restaurantes de comida rápida se espera que sea el propio cliente el que lleve la hamburguesa hasta el expositor de guarniciones, se sirva él mismo la bebida y retire eficazmente los restos de alimentos y la bandeja una vez concluya, como ejemplo de imposición de tareas al consumidor — desviación de objetivos- que se han multiplicado sin parar.

\section{El desplazamiento de objetivos y sus disfunciones}

Al hilo de lo anterior, es básico conocer cuáles son los objetivos reales en contraposición a los oficiales o «declarados» en los estatutos de la organización, cómo aparecen, cuáles y cuántos son, cuál es su influencia en la orientación histórica de la organización, cómo influyen y son influidos por el entorno, etc. (Delcourt, 1973). En el caso de la organización productiva, se daba por supuesto que el único objetivo empresarial era la productividad y, por ende, el principal problema objeto de investigación era cómo los trabajadores se ajustarán a ese objetivo. Tanto en el taylorismo, a través de la organización científica del trabajo (OCT), como en la Escuela de Relaciones Humanas (RR. HH.), en sus explicaciones sobre la motivación subyace la idea de que la organización en su conjunto está en función de lograr el máximo rendimiento como objetivo único. En realidad, «lo que no se deja regir por la leyes límpidas de la maximización del beneficio como expresión sublime del racionalismo occidental puede convertirse en fuente de anomia, de caos» (Fernández Steinko, 1999: 36) (imagen 4).

Si bien el planteamiento weberiano sobre la racionalidad de la burocracia había tenido un serio revés con los descubrimientos de la Escuela de Relaciones Humanas, el procedimiento democrático para aumentar la eficiencia administrativa tenía efectos imprevistos que dificultaban su desarrollo. Esta idea está ya implícita ya en Michels (1967) y su «ley de hierro de la oligarquía» a partir de la estructura burocrática de los partidos obreros, es decir, cuando la burocracia pone el énfasis en la disciplina y en la observancia de las reglas, lleva a los funcionarios a concebir los procedimientos formales no como meros medios para alcanzar ciertos objetivos, sino como fines en sí (Blau, 1975). El descubrimiento, por parte de R. K. Merton (1957), de las disfunciones burocráticas, o, lo que es lo mismo, las disfunciones respecto a la administración racional, supone un replanteamiento y un proceso de depuración de la teoría de Weber sobre este asunto. El 
Imagen 4. Desplazamiento de objetivos

\begin{tabular}{|c|c|}
\hline Pensadores y corrientes & Planteamiento \\
\hline $\begin{array}{l}\text { - Organización Científica del } \\
\text { Trabajo (OCT) } \\
\text { Escuela de } \\
\text { Relaciones } \\
\text { Humanas (RR. HH.) } \\
\text { - Wilfredo Pareto }\end{array}$ & $\begin{array}{l}\text { Todo lo que no es racionalismo } \\
\text { individual, es "irracionalidad" social, } \\
\text { en las organizaciones y se aceptamos } \\
\text { esta segunda dimensión, aceptamos } \\
\text { que hay que optar por otros } \\
\text { objetivos, más allá de la } \\
\text { maximización de la producción y los } \\
\text { beneficios (Fernández Steinko, } \\
\text { 1999:36). }\end{array}$ \\
\hline $\begin{array}{l}\text { - Michels (1967) y su "ley de } \\
\text { hierro de la oligarquía" }\end{array}$ & $\begin{array}{l}\text { Cuando la burocracia pone el énfasis } \\
\text { en la disciplina, lleva a los } \\
\text { funcionarios a concebir los } \\
\text { procedimientos formales no como } \\
\text { meros medios para alcanzar ciertos } \\
\text { objetivos, sino como fines en sí, }\end{array}$ \\
\hline - $\quad$ R. K. Merton (1957) & $\begin{array}{l}\text { Las disfunciones burocráticas } \\
\text { inducen las disfunciones respecto a } \\
\text { la administración racional. }\end{array}$ \\
\hline $\begin{array}{l}\text { - La corriente racionalista o } \\
\text { neoracionalista }\end{array}$ & $\begin{array}{l}\text { la racionalidad camufla el poder o su } \\
\text { ejercicio: a medida que las reglas } \\
\text { aumentan, más posibilidades de } \\
\text { desviación (González W, 2003:77) }\end{array}$ \\
\hline
\end{tabular}

Fuente: elaboración propia.

funcionalismo ha dado ejemplos de la divergencia entre los objetivos y el comportamiento «ideal» que debe predominar en los sistemas burocráticos. Son muchos los ejemplos, ya conocidos, de estudios empíricos: en el caso de Selnick (1949), sobre Tenesse Valey Authority, se nos presenta el proceso de implantación de una oficina estatal en un medio hostil y cómo trata de lograr sus objetivos optando por un sistema de cooperación informal, lo cual llega a provocar una revisión de las políticas oficiales. M. Grocier (1963), por su parte, en su estudio del «fenómeno burocrático», en el que, una vez que han sido establecidos los procedimientos finales para abordar los objetivos, el profesional que es capaz de hacer frente al resto de problemas adquiere un poder real mayor que el que se deriva de su posición formal. Sin embargo, como señala Simon (2003), la tesis racionalista es optimista porque subestima el costo de la gestión burocrática de la empresa, es decir, sus efectos disfuncionales. Efectivamente, las reglas que definen las tareas, los recursos disponibles, los canales de comunicación, los límites del ejercicio de la autoridad, la composición y funcionamiento de los grupos, las modalidades de coordinación, etc., no son una condición suficiente de eficacia por sí mismas, al no tener en cuenta la actitud de las personas y los grupos hacia las reglas formales. El reconocimiento de las reglas depende también de su adaptación a las condiciones reales e inmediatas del entorno. Si esto no es así, las normas o reglas envejecen y aparece su inadaptación. En resumidas cuentas, la legitimidad de las reglas depende a la vez del reconocimiento de la legitimidad de los objetivos que se persiguen en la organización y supone una eficacia, y de la legitimidad acordada o reconocida al grupo que ostenta el poder y que ejercita la autoridad. 
La crítica a la corriente racionalista o neorracionalista se basa en que la racionalidad camufla el poder o su ejercicio (González, 2003). Existe un «círculo vicioso burocrático», a medida que las reglas aumentan, más posibilidades de desviación, se necesitan más sanciones, y, como consecuencia, surge una proliferación de nuevas reglas que terminan por ser más paralizantes que eficaces. De aquí el interés por investigar la «fidelidad» de los grupos, la identificación suficiente del personal a la empresa, a los objetivos (rentabilidad y beneficios), la motivación, la participación y formación de personal. Para el funcionalismo relativo la empresa y las organizaciones están constituidas por constelaciones de grupos, redes de influencia internas y externas, que se transforman en fuerzas sociales diversas que evolucionan y cambian en función de las condiciones del entorno.

Para la corriente realista no hay más que solidaridades limitadas y cambiantes, racionalidades parciales difíciles de combinar, teniendo como consecuencia que la jerarquía de los objetivos no puede ser más que momentánea. La empresa no es más que un conjunto de esfuerzos estructurados y de fuerzas canalizadas. Toda solución es susceptible de poder ser puesta en cuestión. La organización está siempre en una suerte de equilibrio inestable, en la medida en que una persona o grupo toma una decisión corre el riesgo de crear en otros una sucesión de problemas, y sea este proceso el que incentive a bloquear una solución.

\section{La racionalidad y la toma de decisiones}

La aportación de Simon a la «teoría de la organización» hay que enmarcarla en la corriente neorracionalista, que puede ser considerada como un intento de rellenar el vacío existente entre los extremos de las teorías organizacionales del comportamiento, es decir, el empírico donde los psicólogos sociales de la Escuela de las RR. HH. descubren los aspectos «irracionales» del comportamiento, y el normativo donde los economistas y los teóricos de la OCT no solo sitúan a la racionalidad en la cúspide de la organización, sino que olvidan también los aspectos no racionales del comportamiento (Mouzelis, 1973; Fernández Steinko, 1999).

$\mathrm{Al}$ igual que ocurre en los aspectos del comportamiento estudiados por la psicología relativos al aprendizaje, la percepción, etc., el comportamiento administrativo se basa en la máxima de que los organismos se comportan de modo consecuente al medio en el que se desenvuelven, en la medida en la que la complejidad aumenta, su capacidad de tratamiento de la información y cálculo se ven reducidos. La explicación está en que los procesos de información, individuales y organizacionales se rigen por leyes de percepción selectiva, y en consecuencia los decisores tienen reacciones diferentes ante los acontecimientos. Asimismo, los procesos de planificación en la administración de la organización son idénticos 
a los de la psicología experimental en la solución de los problemas individuales. Pero además hay que tener presente que el criterio de satisfacción constituye uno de los medios principales para detectar el proceso de selección de decisiones con las limitaciones del proceso de información. El nivel de aspiración se regula a lo largo del tiempo en función de la facilidad o dificultad del individuo para encontrar alternativas satisfactorias (Simon, 1974) (imagen 5).

Imagen 5. Racionalidad y toma de decisiones

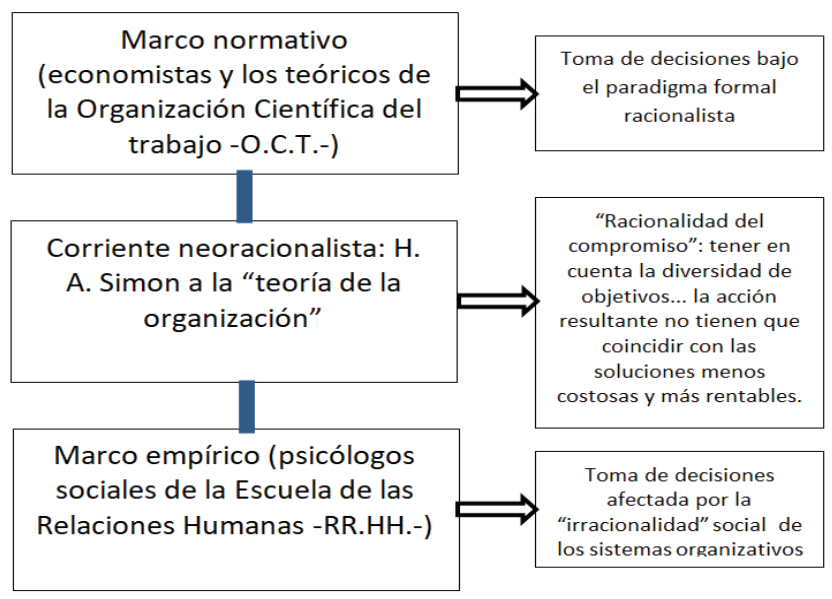

Fuente: elaboración propia.

Dados los límites de la racionalidad en la toma de decisiones en las organizaciones, se establecen dos premisas: «las premisas fácticas» (conocimiento completo de las alternativas y sus consecuencias), cuya validez hay que someterla a verificación empírica y hacen referencia a la elección de los medios, y «las premisas de valor» (utilidad u orden de referencia), no sujetas a verificación, y conciernen a la elección de los fines de la organización. Estas premisas no vienen dadas, los actores que toman las decisiones tienen que buscarlas, y es en el proceso de búsqueda donde existe toda clase de limitaciones reductoras de la calidad y cantidad de la información en las que se basan las decisiones (Mouzelis, 1973).

Tanto March y Simon (1958) como Etzioni (1961) formulan el concepto de la racionalidad en las organizaciones como «racionalidad del compromiso»o «racionalidad limitada». Rechazan la idea de una «racionalidad neutra», ya que ante la confluencia de grupos de interés esta solamente puede ser una «racionalidad negociada» (Delcourt, 1973). Por eso, la corriente neorracionalista no contradice el análisis weberiano, sino que ofrece una explicación de fenómenos que no forman parte del «ideal-tipo» de las organizaciones de racionalidad burocrática 
e instrumental. Si bien la «racionalidad del compromiso» no es el ideal, sí es un «camino practicable», al tener en cuenta la diversidad de objetivos, de personas, grupos y departamentos. Se entiende, por tanto, que la decisión y la acción resultantes del compromiso no tienen que coincidir con las soluciones menos costosas y más rentables. Se impone la idea de una racionalidad establecida paso a paso, «gradual», en términos de Etzioni, sería una racionalidad que arranca de un conocimiento limitado de los factores y productos que se pueden controlar; la rentabilidad depende de la actitud que toman los grupos y las reacciones del entorno a estos pequeños cambios. Es la experiencia de los cambios limitados la que permite la eficacia de un cambio de rumbo o un crecimiento importante. El comportamiento o las decisiones racionales son alcanzados a partir de correcciones y de ajustes sucesivos. El «realismo administrativista» no busca soluciones «óptimas», sino salidas «viables» (no lo «máximo», sino lo «factible») (Hidalgo Tunón, 1978). Simon utiliza la denominación «racionalidad sustantiva» para referirse al concepto de racionalidad surgido dentro de la economía; y «racionalidad procesual» para referirse al concepto de racionalidad utilizado en psicología: «el comportamiento es intencionalmente racional cuando es aceptado para alcanzar metas dentro de los límites impuestos por las condiciones y las restricciones dadas». De acuerdo con esta definición, la racionalidad depende de las metas, una vez establecidas, el comportamiento racional está determinado enteramente por las características del ambiente (Hahn y Hollis, 1986; Suárez, 1992).

La aportación más importante de esta subcorriente está en el análisis de las políticas que corresponden a los imperativos funcionales de la organización y las racionalidades parciales de los grupos y de los hombres en el seno de la organización. Al mismo tiempo, los neorracionalistas examinan cómo y sobre qué puntos el entorno turbulento afecta en todo o parte al funcionamiento y al desarrollo de la organización. Para estos autores, la organización hace frente al entorno externo e interno readaptando sus políticas y sus acciones, desviando sus objetivos y forzándola a definir y redefinir sus políticas. Al mismo tiempo, estas políticas tienen necesidad de tener en consideración las diversas líneas de acción de los actores.

Jeremy Rifkin (2014) relata el momento en el que la estandarización eficaz de la producción en serie del Ford $\mathrm{T}$ dio lugar en los años veinte del siglo pasado a la producción en masa, estableciendo las normas que posteriormente habrían de regir este tipo de actuación industrial durante más de cincuenta años. Según un estricto estilo tayloriano, las fábricas de Ford y del resto de productores de Detroit se organizaban en rígidas líneas jerárquicas, con una estructura de mando que iba de la parte superior de la organización a la inferior. La masa laboral encargada del montaje de los Ford T carecía de cualquier conocimiento especializado y estaba excluida de cualquier tipo de control sobre el ritmo de la producción. 
En los años veinte Ford fabricaba dos millones de vehículos al año, todos idénticos. Refiere Rifkin como en cierta ocasión Ford comentó que sus clientes podían escoger para su Ford T cualquier color que quisiesen, siempre que fuese negro. Este sistema de producción industrial se expandió a otros sectores, ya hemos comentado el concepto de macdonalización de la sociedad formulado por Ritzer (1993). Pero, mientras el modelo de producción norteamericano se extiende con éxito por todos los sectores y parte del mundo, una empresa japonesa, después de la Segunda Guerra Mundial, con métodos diferentes a los de la producción en masa, comienza a lograr objetivos similares con procedimientos diferentes, era Toyota y su proceso de trabajo se denominaba «producción racionalizada». Rompiendo con el modelo taylorista, esta nueva forma de racionalidad organizativa parte de desechar las formas tradicionales de jerarquía organizativa y las sustituye por equipos multidisciplinares que trabajan conjuntamente en los puntos de producción. La consecuencia posterior es que «los planteamientos operativos de la gestión racionalizada, con el importante énfasis en el concepto de "proceso" en lugar de "estructura y función", han hecho que los fabricantes japoneses están preparados para obtener ventajas de las nuevas tecnologías de la información basadas en los ordenadores» (Rifkin, 2014:208).

\section{Racionalidad de los agentes y de los sistemas}

La progresiva complejidad tecnológica de la empresa mantiene una importante correspondencia con los cambios que se operan en su organización. La evolución de la racionalidad de los agentes, por tanto, en cierto modo se correspondería con la adaptación del «sistema» a los nuevos «inputs» tecnológicos. En expresión de Touraine (1965), la evolución de la técnica y su correspondencia con lo organizativo y social se producirá por fases, dialécticamente concebidas: la fase A, presupone la autonomía en el oficio (sistema profesional), que se iría debilitando de forma progresiva al avanzar la automatización (fase $\mathrm{C}$ o sistema técnico), y entre ambas mediaría en período transitorio (fase $B$ o trabajo en cadena), que negaría importantes aspectos del estadio A al tiempo que anunciaría otros del C. En coherencia con esta idea, Woodwar (1965) sostiene que las empresas diseñan sus estructuras formales ajustándolas al tipo de tecnología empleada. Es evidente que el factor tecnológico es decisivo a la hora de adoptar decisiones de carácter organizativo, y en el siglo xxı, más decisivo si cabe que factores como el mercado, la diversidad de productos, el tamaño de las empresas, etc. El grado de complejidad tecnológica debería guardar una relación «lineal» con la amplitud del personal sometido a los directores, la proporción de personal directivo y administrativo, es decir, el número de niveles de autoridad. En cambio, la relación debería ser 
«curvilínea» en otros aspectos, mientras que en la producción de grandes series son aceptables una mayor burocratización y un sistema de dirección más bien rígida (o mecánica), en otros casos se impone una funcionalidad de estructuras organizativas flexibles.

Los sistemas de fabricación han logrado hacer compatibles los requerimientos de flexibilidad y productividad con capacidad para adaptar fácil y rápidamente el proceso productivo a los cambios de la demanda (Escudero Zamora, 1987), ya se trate de cambios cualitativos (nuevos productos) o cuantitativos (número de unidades físicas producidas). Al mismo tiempo, las necesidades de la descentralización productiva ("producción difusa») determinada por nuevas formas tecnológicas suponen una reorganización o «sistema de fábrica» abierto, con la flexibilidad y descentralización como valores centrales (Antona, 1981).

Ahora bien, Atkinson (2015), en su referencia a la obra de Harry Braverman, señala que tanto Marx como Weber predijeron que el auge de la tecnología en la industria capitalista vendría acompañado de elevados niveles de eficiencia, y que la racionalización de la mano de obra, aunque en realidad del conjunto del sistema productivo, derivaría en tareas más especializadas y diferenciadas. Braveman, continúa indicando Atkinson, no pretende el retorno al modelo preindustrial; y reconoce que la automatización y la racionalización pueden ser positivos. Pero defiende que sus efectos se tornan negativos cuando van acompañado de cambios radicales en las relaciones sociales de producción. Esta es la base de la crítica de la racionalidad instrumental, científica y tecnológica, en la cual, bajo el paraguas del sistema científico y técnico capitalista, lo irracional parece tornarse razón (Marcuse, 1968). Por ejemplo, la apropiación que las organizaciones hacen de la ciencia $(\mathrm{el} \mathrm{I}+\mathrm{D}+\mathrm{i})$ no engendra simplemente el crecimiento, también sobreproducción, despilfarro, desempleo masivo e inseguridad laboral. De esta forma, estamos ante una de las principales paradojas de las sociedades altamente tecnificadas: «el carácter racional de su irracionalidad». Este sistema es capaz de incrementar y de generalizar el confort, de hacer superflua una necesidad, de presentar la destrucción constructiva; pero al mismo tiempo transforma el mundo en objeto, en una dimensión del cuerpo y del espíritu humano, de modo que la misma noción de alienación se transforma en problemática. Las personas se identifican con sus mercancías, encuentran su alma en el automóvil, en Spotify, en el «Internet de las cosas». Ahora bien, que la realidad haya absorbido a la ideología no significa que no haya más ideología. Al contrario, la racionalidad industrial es más ideológica que aquella a la que precedió, porque la ideología está en el mismo el proceso de producción. Para Marcuse (1968), en la realidad social, a pesar de todo cambio, la dominación del hombre por el hombre es un continuismo histórico, hay todavía un lazo histórico entre la razón pretecnológica y la razón tecnológica. Entretanto, 
la sociedad que concibe y que emprende la transformación de la naturaleza por la tecnología cambia los principios de base de la dominación. La dependencia personal (aquella que liga al esclavo con el amo) ha sido reemplazada por otra clase de dependencia, aquella que está ligada al «orden de cosas objetivo» (las leyes económicas, el mercado, etc.). El hecho de que el hombre se encuentre progresivamente encadenado al aparato productivo revela los límites de esta racionalidad y su fuerza siniestra, este aparato productivo perpetúa la lucha por la existencia, y tiende a una concurrencia internacional total que compromete la vida de aquellos que han construido ese aparato y se sirven de él.

La racionalidad «procesual» de Simon, principalmente en el proceso de toma de decisiones, aparece en el contexto de la conducta; así, la economía necesita comprender cómo las personas se comportan ante la incertidumbre, y qué límites de información y computabilidad les acompañan (González, 2003). Si los sistemas tecnológicos han sido valorados como un Deus ex machina, estos se ubican en un sistema social que les da sentido, y que juega a favor de la racionalidad y de los intereses de los dominantes.

Godelier (1976) nos da la oportunidad de realizar un planteamiento de tipo sociológico, en el sentido de que la génesis histórica del principio formal de la racionalidad, que es la acción orientada a un fin, no supone regresar a las posiciones que ven en dicho principio una propiedad de la naturaleza humana, pues la universalidad de este principio nada explica de la diversidad del contenido de la actividad humana, ni de las razones de la aparición y la desaparición en la historia de los sistemas económicos y sociales. Dentro de la dinámica que impone el «nuevo» sistema de globalización, la falsa racionalidad, esto es, la racionalización abstracta y unidimensional, se hace hegemónica (Morín, 1993).

\section{La dinámica organizacional: entre la racionalidad limitada y el desplazamiento de los objetivos}

De un lado, tenemos el establecimiento de diferentes criterios de racionalidad (sobre todo económica y técnica) y la dificultad de establecer objetivos a través del tiempo a partir de condiciones que cambian y evolucionan constantemente, junto con la necesidad de medir el grado de incertidumbre apuntada por Thompson (1967), lo cual nos conduce a lo que se ha dado en llamar «la teoría ecológica de las organizaciones» (Hannan y Freeman, 1989), como un modelo que puede integrar estos tres factores. Uno de los planteamientos macrosociológicos más originales sobre las organizaciones es el de Aldrich (1989), el cual analiza, desde el punto de vista empírico, «agrupaciones» de diferente índole (poblaciones de empresas de un sector, organizaciones políticas o sociales), llegando a 
la conclusión de que las organizaciones nacen, se desarrollan y muchas de ellas mueren. Los procesos (variación, selección y retención) que subyacen en estos movimientos naturales se repiten ad infinitum a lo largo del tiempo. Si bien sigue la tradición de los investigadores que propusieron modelos de «ciclo vital» de las organizaciones (Chandler, 1962; Greiner, 1972), describiendo los cambios que las organizaciones sufren a medida que evolucionan y las repercusiones que ello tiene en los planteamientos relativos a su administración, planificación y control.

En ambas propuestas, la de la ecología organizacional y la del ciclo vital, el fin principal es explicar por qué ciertas formas organizativas sobreviven y proliferan en tanto que otras languidecen y desaparecen (Gómez Llera, 1994). Se trata de enfatizar que el proceso de selección no depende necesariamente de los presupuestos de optimización o eficiencia, sino que puede ser provocado por una gran variedad de factores. Según Hannan y Freeman (1989), existe una selección natural, solo subsisten las organizaciones equipadas adecuadamente para el cambio. Aunque los líderes puedan ser personas muy activas, con metas y objetivos muy claros, las organizaciones que se resisten a cambiar sus estructuras, roles, redes de autoridad y sistemas de comunicación tienden a desaparecer (Rodríguez, 1991: 208) (imagen 6).

\section{Imagen 6. La dinámica organizacional: el entorno ecológico}
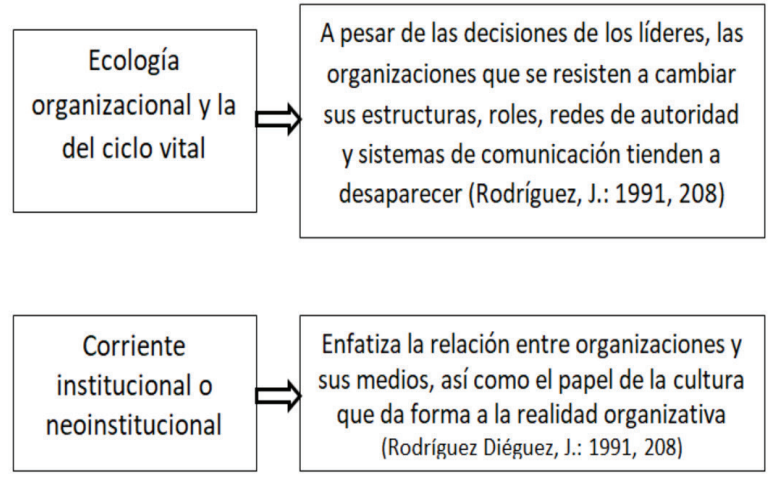

La «corriente institucional o neoinstitucional», representada por Meyer y Rowan (1977) y DiMaggio y Powell (1983; 1990), incorporó los elementos de la cultura de la organización (símbolos, sistemas de creencias, reivindicaciones y programas profesionales) que están o que inciden en esta dinámica. Según lo cual las organizaciones compiten no solo por recursos y clientes, sino también por poder político y legitimidad institucional. Esta perspectiva aleja su atención de la preocupación por los recursos, la existencia de clientes o el número de competidores, para centrar su atención en el papel que juegan, por ejemplo, el Estado, el 
sistema legal y las profesiones en el proceso de dar forma a la vida organizativa, tanto directamente como con la generación de nuevos «mitos racionales». Los institucionalistas mantienen que el motor de la racionalización y burocratización ha pasado del mercado al Estado y a los profesionales (Rodríguez Diéguez, 1991). La institucionalización es un proceso que depende del Estado y que convierte a las organizaciones en menos operativas, al limitar las opciones que pueden seguir. Enfatiza la relación entre organizaciones y sus medios, así como el papel de la cultura dando forma a la realidad organizativa.

\section{Conclusión: flexibilidad y adaptabilidad dentro del sistema}

Los enfoques anteriormente descritos, y a pesar de plantear una consideración explícita de las organizaciones como sistemas abiertos compuestos por un subsistema social y otro técnico, y la relación con el entorno, no dejan de ser un planteamiento estratégico al tratar de adaptar la empresa a las necesidades sociales a través de decisiones estratégicas. En estas perspectivas, la empresa no es concebida como un sistema formal (con reglas, normas y funciones), sino como una institución que evoluciona según las necesidades sociales. Es decir, la empresa imbuida de una racionalidad social a partir de intereses divergentes, y en relación con los elementos del medio ante el que adopta una estrategia que se convierte en táctica (el «día a día») o bien como consecuencia de una competencia imperfecta (de carácter oligopólico) que la somete a turbulencias y a un sistema de decisiones dependiente del sistema económico en el que se encuentra inmersa y de la relaciones sociales que aquel implica (Erbes-Seguin, 1972). El caso es que, en todos estos planteamientos, la gestión empresarial bascula en un continuo que va desde el cálculo racional estricto hasta la arbitrariedad. Como señala Prieto (1993), el grado de acercamiento a la racionalidad determinará la mayor o menor viabilidad económica de la empresa en coexistencia con distintos niveles y formas de arbitrariedad. En la empresa, como organización productiva, si bien es cierto que hay que tomar en cuenta su sistema técnico, su sistema de relaciones informales, su organización burocrática, que tiende a fijar estatus, roles y relaciones de trabajo, su sistema de decisiones, teniendo en cuenta el entorno y los intereses de los grupos de actores, desde un punto de vista epistemológico, no hay que olvidar que los condicionantes de la estructura, de las decisiones y de las relaciones sociales en su interior se encuentran en la dinámica de un sistema socioeconómico en un momento histórico con «un estilo de desarrollo» dominante que se debe tener en cuenta a partir de una perspectiva amplia de análisis. 
La idea esencial que subyace en este artículo es que las diferentes perspectivas que enfrentan el estudio de la racionalidad administrativa es un capítulo que no está aún cerrado, ni prefigurado cuál será su desarrollo en la sociedad hiperconectada y superdigitalizada del siglo xxI, como evidencia el ejemplo de la macdonalización, por un lado, en el que se describe el desbordamiento, incluso el desplazamiento de los objetivos, y lo que podríamos calificar de disfunciones, generadas desde estas organizaciones, para lo cual es necesario encontrar marcos conceptuales suficientemente explicativos, o adaptar adecuadamente los existentes. De manera complementaria, los diferentes esquemas de racionalidad, tanto en los procesos de toma de decisiones como en la ordenación de las estructuras internas de las organizaciones, ponen de manifiesto, a partir del caso de Toyota, la ruptura de una cierta comprensión de racionalidad organizativa asociada, en parte, esencialmente, a la concepción weberiana y sus derivados. $Y$ conecta con los desarrollos de Simon en cuanto a los límites de esta racionalidad, o más bien la diversidad y ductilidad de las variadas formas que adopta.

\section{Bibliografía}

Aldrich, Howard E. (1989). Organizations and Environrnents. Englewood Cliffs: Printice-Hall.

Atrinson, Sam (2015). El libro de la sociología. Barcelona: Akal.

Bauman, Zygmunt (2003). Comunidad. En busca de seguridad en un mundo hostil. Madrid: Siglo XXI de España Editores.

Beck, Ulrick (2009). La sociedad del riesgo. Hacia una nueva modernidad. Barcelona: Paidós Surco.

Bilbao, Andrés (1996). La racionalidad económica y la secularización. Revista Española de Investigaciones Sociológicas, 74, 225-243.

Blau, Peter (1975). "A Formal Theory of Differentiation in Organizations". American Sociological Review, 35, 201-218.

Brunet Icart, Ignasi y Catalin Mara, Liviu (2018)."Las organizaciones, en Iglesias de Ussel, Trinidad Requena y Soriano Miras". En La sociedad desde la sociología. Una introducción a la sociología general. Madrid: Tecnos.

Bude, Heinz (2017). La sociedad del miedo. Barcelona: Herder.

Chandler, Alfred (1962). Strategy and structure. Cambridge: MIT Press.

Delcourt, Jean (1973). Sociologie du travail. Mineo: Université Catholique de Louvain.

Erbes-Seguin, Sabine (1978). Sociología del Trabajo. Madrid: Pirámide. 
Escudero Zamora, Gabino (1987). “Tecnología y sistema productivo: cualificación y descualificación". En J. M. Garmendia. Sociología industrial y de la empresa. Madrid: Aguilar.

Etzioni, Amatai (1962). Complex Organizations: A Sociological Reader. New York: Holt, Rinehart \& Winston.

Fernández Steinko, Armando (1999). "Sociología y teoría económica: cien años de indeseable desencuentro". En C. A. Castillo Mendoza. Economía, organización y trabajo. Un enfoque sociológico (pp. 19-46). Madrid: Pirámide.

Furtado, Celso (1982). El subdesarrollo latinoamericano. Méxicoः FCE.

Garmendia, José Antonio (1988). "La cultura de empresa. Una aproximación teórica y práctica”. Revista Española de Investigaciones Sociológicas, 41, 7-23.

Garmendia, José Antonio (1990). Desarrollo de la organización y cultura de la empresa. Madrid: ESIC.

Giddens, Antony (2010). Sociología. Madrid: Alianza Editorial.

Godelier, Maurice (1975). Racionalidad e irracionalidad en la economía. Madrid: S. XXI.

Gómez Llera, Germán (1994). "La adaptación de las organizaciones en su entorno". En A. Lucas Marín (2002). Sociología para la empresa. Madrid: McGraw-Hill.

González, Wenceslao (2003). "Racionalidad y economía: de la racionalidad de la economía como ciencia a la racionalidad de los agentes económicos." En W. González. Racionalidad, historicidad y predicción en H. A. Simon. A Coruña: Netbiblo.

Greiner, Larry (1972). "Evolución y revolución a medida que crecen las organizaciones". Harvard Business Review, julio-agosto.

Hahn, M. Hollis (1986). Filosofía y teoría económica. México: FCE.

Hannan, Michael (1984). "Structural Inertia and Organizational Change". American Sociological Review, 49, 149-164.

Hannan, Michael (1989). Organizational Ecology. Cambridge: Harvard University Press.

Hannan, Michael (1977). The Population Ecology of Organizations. American Journal of Sociology, 82, 929-966.

Hidalgo Tuñón, Alberto (1978). "El principio de racionalidad limitada de $\mathrm{H}$. A. Simon y el premio nobel de economía”. El Basilisco, 68-79.

Jiménez Blanco, José (1978). Teoría sociológica contemporánea. Madrid: Tecnos. López Novo, Joaquín (1994). “El particularismo reconsiderado. Orientación de la acción y contexto institucional"' Revista Española de Investigaciones Sociológicas, 67/94, 31-63.

López Pintor, Rafael (1986). Sociología Industrial. Madrid: Alianza. 
March, James y Simon, Herbert (1958). Organisations. New York: Wiley.

Marcuse, Herbert (1968). L’homme unidimensionnel. Paris: Ediciones Minuit.

Martinelli, Alberto (1985). "Análisis económico y análisis sociológico en el sistema teórico de Schempeter". Revista Española de Investigaciones Sociológicas, 30, 41-68.

Merton, Robert (1957). Social theory and social structure. México: FCE.

Michels, Robert (1967). Political Parties. New York: Free Press.

Motтеz, Bernard (1971). El salario a rendimiento. El obrero estimulado. Barcelona: Nova Terra.

Mouzelis, Nicos (1973). Organización y burocracia. Barcelona: Ediciones Península.

Pérez Rubio, José Antonio (1997). "Motivación y Satisfacción laboral: retrospectiva sobre sus formas de análisis". Revista Española de Investigaciones Sociológicas, 80 .

Pérez Vilariño, José (1987). "Racionalidad y control en las organizaciones complejas". Revista Española de Investigaciones Sociológicas, 39, 119-139.

Perrow, Charles (1998). Sociología de las organizaciones. Madrid: McCraw-Hill.

Perrow, Charles (1967). "A Framework for the Comparative Analyses of Organizations". American Sociological Review, 32, 194-208.

Prieto Rodríguez, Carlos (1993). "Límites de la racionalidad económico-mercantil en la relación salarial". Revista Española de Investigaciones Sociológicas, 65, $53-70$.

Rifkin, Jeremy. (2014). El fin del trabajo. Nuevas tecnologías contra puestos de trabajo: el nacimiento de una nueva era. Barcelona: Paidós Booket.

Sastre, Cayo (2010). McMundo. Un viaje por la sociedad de consumo. Barcelona: Libros Del Lince.

Simon, Herbert (2003). "La racionalidad limitada en ciencias sociales: hoy y mañana”. En W. González. Racionalidad, historicidad y predicción en H. A. Simon. A Coruña: Netbiblo.

Simon, Herbert (1960). "The new science of management decision." Ford Distinguished Lectures, 3.

Thompson, James (1967). Organizations in Action. New Yorkः McGraw-Hill.

Touraine, Alain (1965). "La organización profesional de la empresa”. Friedman y Naville, I, 384-425.

Tuoraine, Alain (1973). La sociedad postindustrial. Barcelona: Ariel.

Villarreal Larrinaga, Oskar (2017). "Is it desirable, necessary and possible to perform research using case studies?" Cuadernos de Gestión, 17(1), 147-172.

Veroz Herradón, Ricardo (1999). Ecología de las organizaciones. Córdoba: Universidad de Córdoba y Caja Sur. 